\title{
Competencias docentes en educación superior
}

Lina Aleida Alcántar Hernández.

1 auge que actualmente tiene la metodología por competencias en el ámbito de la educación, se debe en parte a los cambios que se han dado en la sociedad, lo cual ha derivado en modificaciones al interior de las instituciones educativas. De acuerdo con lo anterior, en las universidades se aprecian en los siguientes aspectos: 1) los estudiantes son autónomos en la adquisición del aprendizaje, utilizando varias herramientas digitales; 2) la empleabilidad, pues una carrera universitaria ya no es suficiente para obtener un empleo más o menos rápido al egresar; 3) los estándares en la calidad de la educación universitaria; 4) el conocimiento, el cual debe ayudar a la formación de profesionales que se desempeñan en diversos escenarios de trabajo; 5) el profesor ahora debe centrar su actividad docente en el aprendizaje de los estudiantes y no en la enseñanza. Como resume Zabalza (2006), existen cambios en tres niveles: el escenario institucional, la estructura del conocimiento y el papel a desarrollar por el profesor.

A lo mencionado se suma el desarrollo tecnológico, el cual da lugar a nuevas metodologías y roles docentes (Fernández, 2003), teniendo como consecuencia que el perfil docente deba redefinirse para describir las funciones que el profesor realiza en la actualidad.

Como ya se mencionó, el principal cambio del docente es su rol; él ya no es la fuente del conocimiento, ahora es el guía facilitador de información, recursos y estrategias de aprendizaje (Peón e Ibarra, 2004), convirtiéndose en un gestor de información y orientador de experiencias de aprendizaje.

Fernández (2003) expone que las principales funciones que los profesores realizan actualmente son: 1) planificar cursos; 2) diseñar estrategias para la enseñanza y el aprendizaje; 3) buscar y preparar recursos y materiales didácticos; 4) gestionar la información y el desarrollo de las clases para mantener el orden; 5) motivar al estudiante; 6) estimular la participación de los estudiantes; 7) facilitar la comprensión de los contenidos básicos; 8) ser ejemplo de actuación y portador de valores; 9) asesorar al estudiante para el uso de recursos; 10) orientar la realización de las actividades; 11) tutorar; 12) trabajar directamente con los estudiantes; 13) evaluar; 14) fomentar actitudes necesarias en la sociedad de la información; 15) realizar trabajos de gestión; 16) actualizarse continuamente; y 17) mantener contacto con el entorno.

Osorio (2011) hace una comparación que proponen diversos autores sobre los rasgos del perfil ideal del docente en educación superior. En la siguiente tabla, se evidencian las funciones que se espera los profesores de este nivel educativo realicen como parte de su quehacer docente, ahora como facilitadores del aprendizaje. 


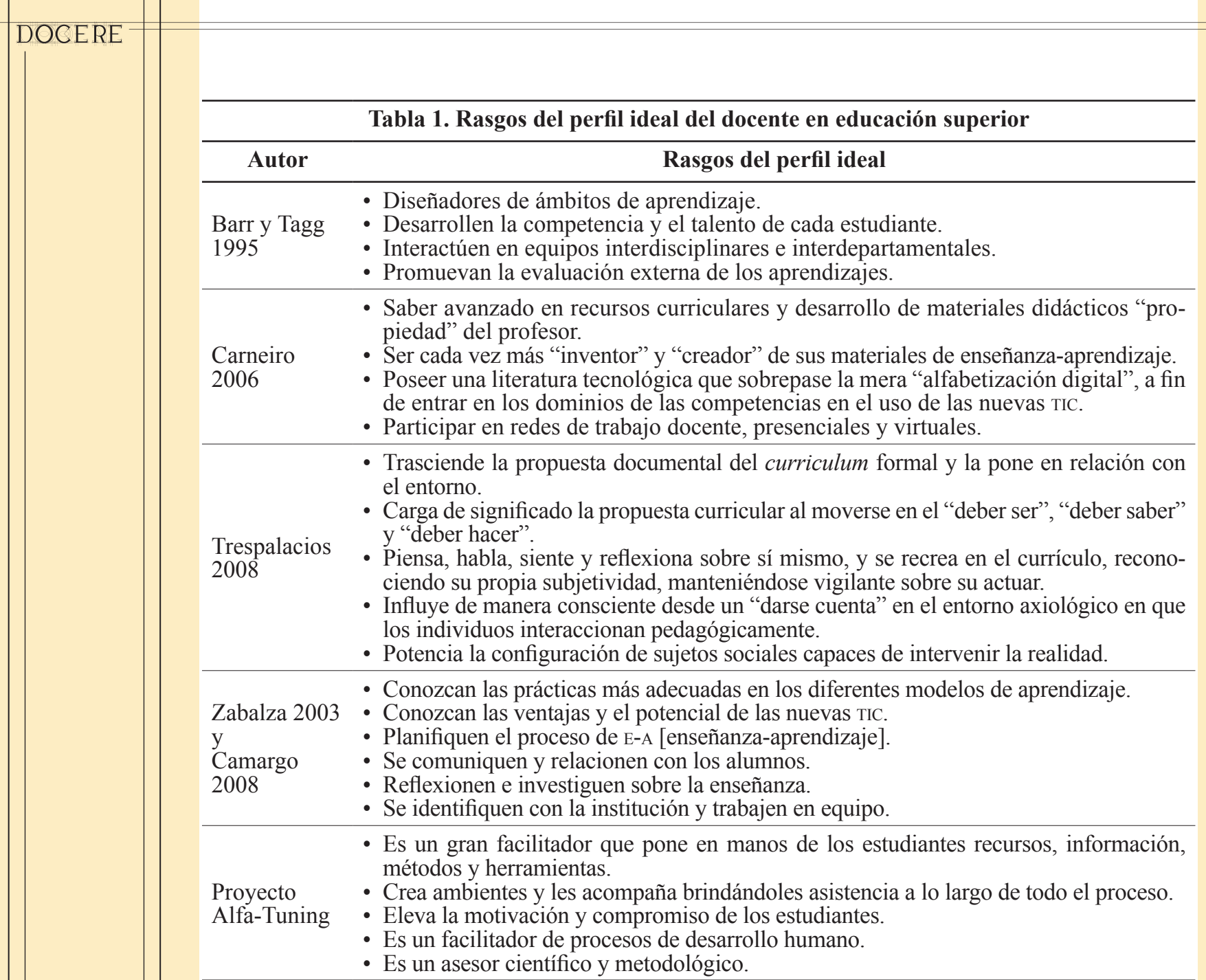

Fuente: Osorio (2011: 152).

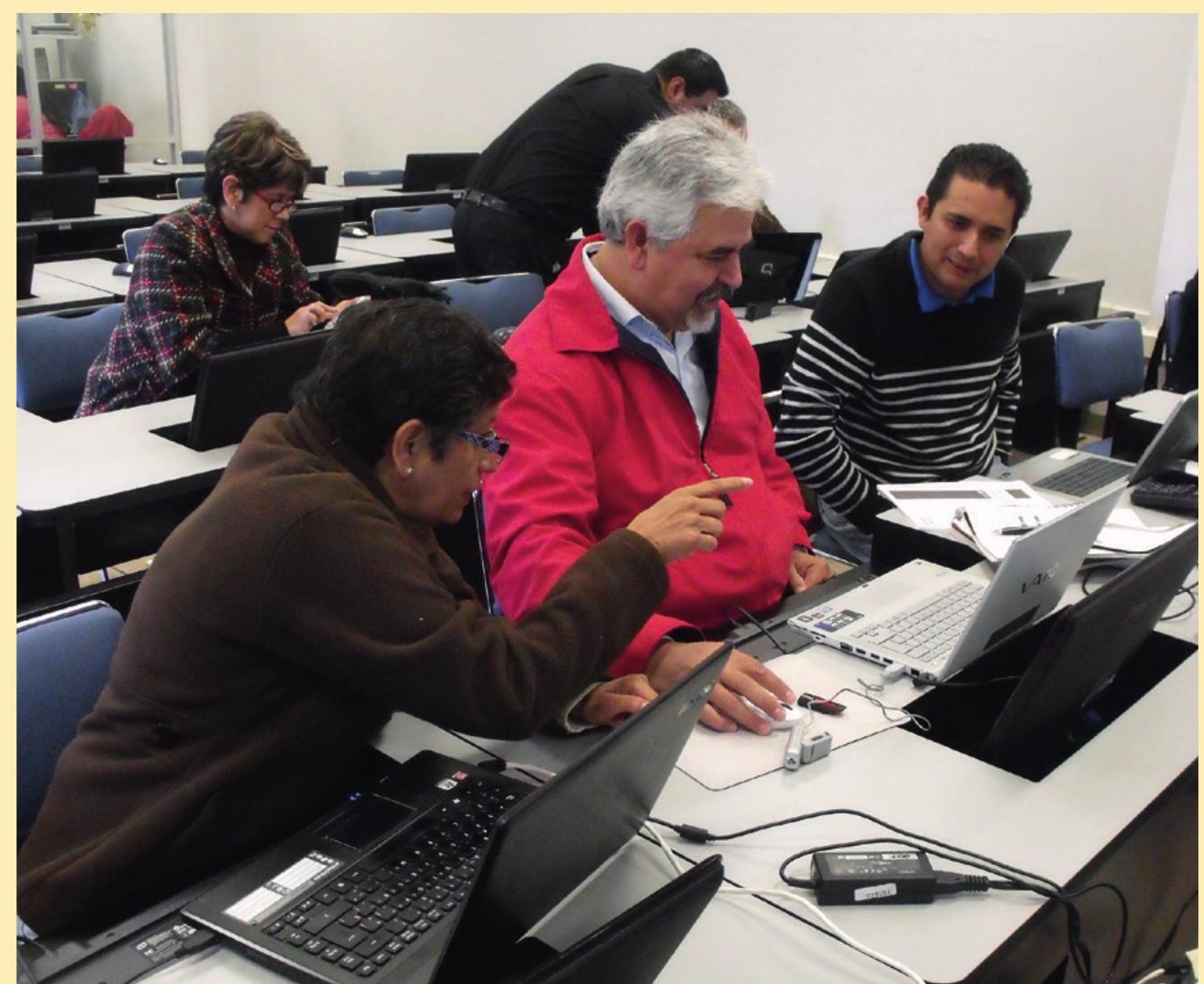




\section{Competencias y saberes docentes}

Dentro del estudio de las competencias docentes, éstas se clasifican en competencias profesionales, básicas y específicas, las cuales son descritas en este apartado:

Competencias profesionales: son exclusivas del profesorado universitario y se definen por Bozu y Canto (2009: 91) como "el conjunto de conocimientos, habilidades, actitudes y valores necesarios para realizar una docencia de calidad. Esto es, lo que han de saber y saber hacer los profesores para abordar de forma satisfactoria los problemas que la enseñanza les plantea".

Competencias básicas: algunos autores como Bozu y Canto (2009) consideran que los profesores requieren una serie de competencias denominadas básicas para realizar con éxito su trabajo docente, como las:

- Cognitivas, específicas de la disciplina que imparte.

- Metacognitivas, para la reflexión de su propia enseñanza, que le permitan mejorar de forma sistemática y continua.

- Comunicativas, para mantener un diálogo constante con los estudiantes y colegas.

- Gerenciales, para la gestión eficiente de la enseñanza y los recursos en diferentes ambientes de aprendizaje.

- Sociales, que le permitan interactuar con los estudiantes de forma asertiva.

- Afectivas, que faciliten la empatía con los alumnos.

- Pedagógicas y didáctico-metodológicas.

Competencias especificas: son aquellas competencias necesarias para cubrir las actividades del perfil docente actual:

- Conocimiento del proceso de aprendizaje del estudiante en contextos académicos y naturales.

- Planificación de la enseñanza y de interacción didáctica.

- Utilización de métodos y técnicas didácticas pertinentes.

- Gestión de interacción didáctica y de las relaciones con los estudiantes.

- Evaluación, control y regulación de la propia docencia y del aprendizaje.

- Gestión de su propio desarrollo profesional como docente.

Los saberes docentes necesarios para el ejercicio educativo propuestos por Tremillo (2011) se resumen en:

1. Saber ideológico, el cual implica compromiso a escuchar y tomar decisiones conscientes, disponibilidad al diálogo, y ejercicio pleno y justificado de la autoridad.

2. Saber científico, pues la enseñanza exige investigación, rigor metódico, asumir lo nuevo, y rechazo a la discriminación.

3. Saber de la condición humana, que demanda al docente tener la conciencia de inacabamiento del conocimiento y de la persona, respeto a la autonomía del estudiante, convicción de que el cambio es posible, y reflexión sobre la práctica docente. 
Como conclusión, la tarea del docente implica poner en práctica las competencias y los saberes docentes, definidos como: "elementos cualitativos necesarios para una cultura democrática, coherente con acciones críticas, dimensiones y/o valores" (Tremillo, 2011: 270). Sin embargo, para que los profesores universitarios adquieran o desarrollen tales elementos, en primer lugar deben estar conscientes de los cambios que sufre el escenario educativo y su propio rol, para así adaptarse al nuevo perfil docente que la educación actual exige.

\section{Fuentes de consulta}

Bozu, Z. y Canto Herrera, P. J. (2009). El profesorado universitario en la sociedad del conocimiento: competencias profesionales docentes. En Revista de Formación e Innovación Educativa Universitaria, 2(2), 87-97.

Fernández Muñoz, R. (2003). Competencias profesionales del docente en la sociedad del siglo XXI. Organización y gestión educativa. En Revista del Fórum Europeo de Administradores de la Educación, 11(1), 4-7.

Osorio Madrid, J. R. (2011). Competencias docentes en la profesión académica en México (146-162). En Jaik y Barraza (Coords.) Competencias y Educación. Miradas múltiples de una relación. México: Instituto Universitario Anglo Español, A. C.

Peón Aguirre, R. e Ibarra A. (2004). Necesidad de nuevas competencias en el docente de educación superior. Recuperado el 30 de diciembre de 2013 en: http://www.uaa.mx/direcciones/dgdp/defaa/descargas/nuevas_ competencias.pdf.

Tremillo González, L. F. (2011). Competencias docentes en la profesión académica en México (240-272). En Jaik y Barraza (Coords.) Competencias y Educación. Miradas múltiples de una relación. México: Instituto Universitario Anglo Español A. C.

Zabalza Beraza, M.A. (2006). La universidad y la docencia en el mundo de hoy. Bogotá: U. Javeriana.

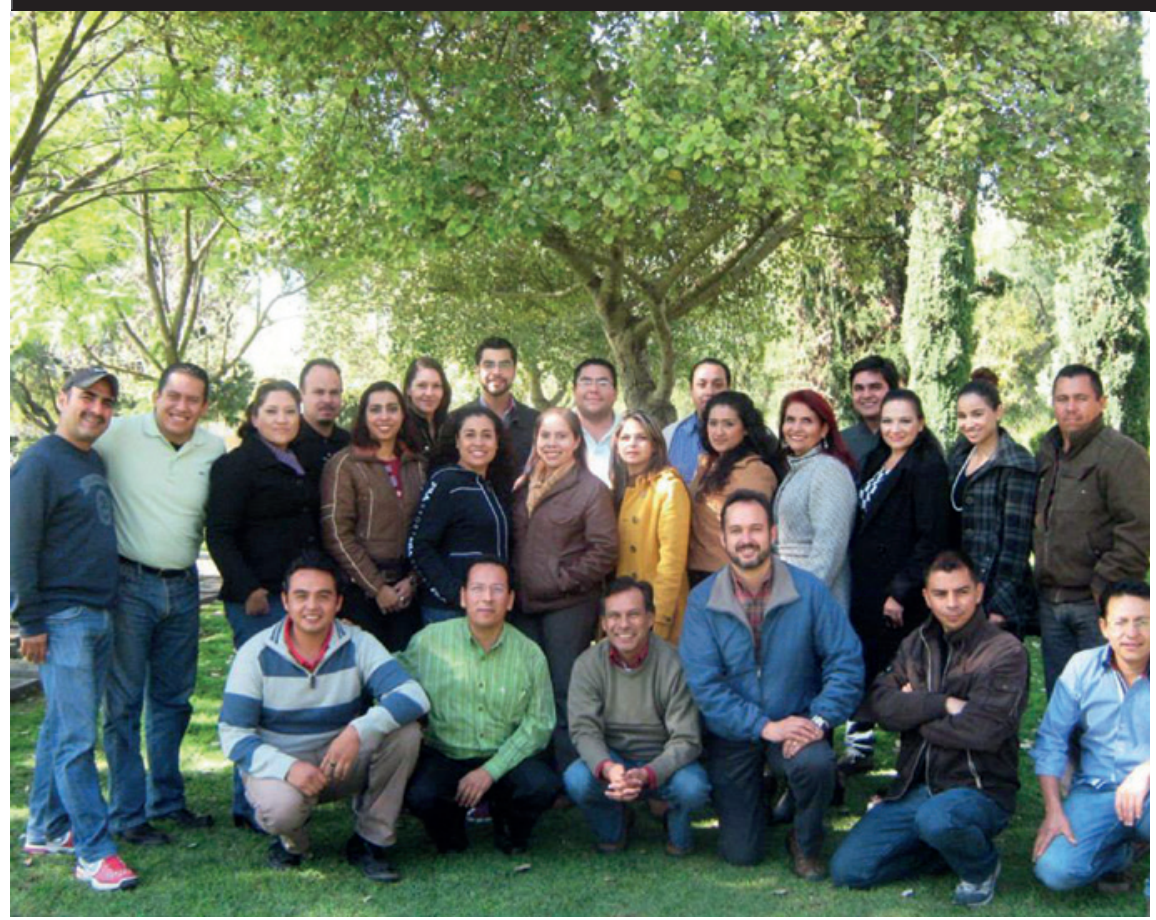

